\title{
LOS ANTIGUOS SEÑORES MIXTECOS DE ACATLÁN, PUEBLA, EN LOS CÓDICES SANCHEZ SOLISS Y TULANE
}

\author{
Arturo Pascual Soto
}

Alfonso Caso, in memoriam.

\section{1. "Sayoo sinica; antiguamente, sin memoria de los presentes"}

En épocas pasadas, los cambios violentos en la distribución del poder político debieron ser cosa frecuente en los pequeños valles ñuiñe (cf. J. Paddok, 1966). Sólo un sistema de intercambio de mujeres, de "alianzas matrimoniales" entre diversos grupos de parentesco, pudo haber asegurado la continuidad de éstos en los propios territorios de la Mixteca Baja.

Es decir, los "matrimonios entre enemigos" debieron disminuir las lu.chas por el dominio del poder, reduciéndolas a escaramuzas ocasionales.

Los valles fértiles pero limitados de la Mixteca Baja probablemente estimularon la inestabilidad política ñuiñe. Si un linaje, un grupo de filiación constituido sobre la base de la descendencia de un antepasado común y las relaciones de parentesco entre sus miembros (cf. G. Balandier, 1976 y R. Fox, 1980), ocupara uno de estos valles tibereños, pocas serían las generaciones en transcurrir antes que el crecimiento del linaje agobiara las tierras del pequeño valle (cf. R. Carneiro, 1970; M. H. Fried, 1967; C. M. Webb, 1973). En estos territorios donde inevitablemente la población creció, la lucha entre vecinos por la posesión de las tierras pudo propiciar la segmentación del linaje e inclusive la "fisión" del grupo original de filiación (cf. R. Fox, 1980). De tal manera que cada segmento confinado del linaje abandonara el pequeño valle y se conformara en oposición a otros como una unidad, aunque fundada en un idéntico principio de descendencia y residencia, con funciones políticas condicionadas por las relaciones mutuas entre los linajes (cf. G. Balandier, 1976).

Al construirse y consolidarse cierta clase de relaciones "ficticias" de parentesco entre los linajes apremiado por la escasa tierra y obligados a escindirse del propio grupo de filiación y residencia, éstas forzosamente derivaron en "casamientos entre enemigos". Si bien el problema habría de continuar, las "alianzas matrimoniales" aliviaron la permanente amenaza de luchas entre linajes vecinos, entre valles colindantes. 
Ahora bien, los linajes se fundan en individuos concretos, los cuales se sitúan dentro de un mismo marco genealógico al sujetarse en forma unilineal a un mismo grupo de descendencia (G. Balandier, 1976). Incorporados como grupos de filiación unilineal podemos encontrar patrilinajes o matrilinajes, dependiendo de que el grupo de descendencia establezca el parentesco entre sus cognados a través de la línea del varón o de la mujer, del padre o de la madre. De tal forma la exogamia, el buscar ma. rido o esposa fuera del propio grupo de parentesco, será algo más que una extensión del "horror" al incesto (cf. R. Fox, 1980) y resultará ser una forma eficiente para regular las relaciones entre distintos grupos de filiación mediante un intercambio de esposos y el contraer "alianzas matrimoniales".

El parentesco "ficticio" entre los miembros de linajes vecinos no logró aliviar en forma indefinida la tirantez política, menos aún cuando dentro de éstos se construían las genealogías reales de los pequeños valles mixtecos. Ocasionalmente, los matrimonios entre miembros de linajes reales enemigos no redujeron suficientemente la probabilidad de una lucha territorial. Una escaramuza sin importancia podía derivar en cambios violentos en el dominio del poder, en un linaje derrotado y la conquista de su territorio, en cambios de la estructura del poder.

En una situación política apremiante, los antiguos linajes mixtecos parecían aferrarse a los "matrimonios entre enemigos" como una única manera de sobrevivir en los valles ribereños ñuiñe (figs. 1 y 2 ).

\section{2. "Chi yo yata; lugar, sitio antiguo de casa"}

Al oeste de Coixtlahuaca y ocupando la porción sur del territorio poblano, el norte de Oaxaca y una parte del oriente de Guerrero se encuentra la Región Nuiñe (fig. 3). A ella se refiere una parte de los códices mixtecos, mencionando entre otros el topónimo "Cerro de la piedra preciosa" o "Cerro de la turquesa". El Códice Sánchez Solís (Codex Egerton 2895), que hace referencia de este lugar, es un documento genealógico pintado en la última parte del siglo XV o en los inicios del XVI y cuyo origen, supone M. E. Smith (1973), probablemente se encuentre en la Mixteca Baja. El signo del lugar "Cerro del tigre y el enrejado" es el de mayor importancia en el documento y a éste se le asocian tres linajes de yaa tnuhu (R. Spores, 1976), de "señores" poseedores del control político. El topónimo probable de la zona de Acatlán (18 $12^{\circ} 6^{\prime}$ "N., 98 $8^{\circ} 3^{\prime} 0^{\prime \prime}$ ) es "Cerro de la piedra preciosa". Como ya lo ha notado M. E. Smith, aparece representado en cuatro ocasiones y relacionado con el signo del lugar "Cerro del tigre y 
el enrejado", en los cuatro casos se acompaña de glosas que pueden contener el nombre mixteco del Jugar y que, escrito en formas distintas, se lee: yoco yoxi dixaa o yoco yoxi tixaha. En el dialecto del Vocabulario en lengua misteca de fray Francisco de Alvarado (1593), vendría a ser: yucu yusi tisaha (cf. M. E. Smith, 1973).

En la "Misteca baxa" y durante los primeros días del mes de enero de 1581, Juan de Vera, entonces alcalde mayor del pueblo de Acatlán, escribió una relación y descripción del poblado y su partido. En ella, incluía un minucioso análisis de la toponimia del lugar:

E1 dicho pueblo de Acatlan en Iengua mexicana se llama asi Acatlan que quiere dezir en la dicha lengua "Lugar de Cañas", porque los me. xicanos que primero llegaron al dicho pueblo hallaron junto a el vn cañaveral grande, y por eso le nonbraron asi sin curar el nonbre que los naturales le tenian puesto en su lengua misteca, en la qual nonbrauan al dicho pueblo Yuctryuxi que buelto en lengua castellana quiere dezir "Serro de piedras preciadas", y asi mismo le llaman el dia de oy en la dicha lengua misteca Yutta tixaa que buelta en lengua castellana quiere dezir "Agua ensenizada", el qual nonbre le pusieron por razon de vn cerro que esta junto a vn arroyo que pasa por el dicho pueblo que se llama "Serro de Seniza", el qual dicho nonbre y del agua que pasa junto a el se conpuso el dicho nonbre de agua ensenizada. Otros nonbres dizen que tiene, de los quales, y del que tenemos dicho en este capitulo que tenia antes que los mexicanos a el viniesen, no ay memoria ni se sabe la razon dellos, y por eso no se pone aqui (Papeles de Nueva España, V:58-9).

Sin embargo, en el año de 1593, fray Antonio de los Reyes sólo registró el nombre mixteco Yutatisaha para el pueblo de Acatlán en su Arte en lengua mixteca. Al parecer, la forma yucu yusi para designar a ese lugar de la Mixteca Baja había sido olvidada (cf. M. E. Smith, 1973).

Ahora bien, fuera del anterior grupo de documentos resulta difícil encontrar una representación toponímica que pueda asociarse a este lugar ñutine sin correr el riesgo de confundirla con la del pueblo chocho de Texupan $\left(17^{\circ} 15^{\prime} \mathrm{N} ., 97^{\circ} 17^{\prime} 0^{\prime \prime}\right.$ ), en la Mixteca Alta, y cuyo topónimo, de tomarse fuera de contexto, comparte los mismos elementos que componen la parte yucu yusi del nombre mixteco conocido para Acatlán (cf. Códice Sierra y el mapa que acompaña la Relación Geográfica de Texupan). Fray Antonio de los Reyes (1593) y la Relación Geográfica concuerdan con el nombre mixteco $\tilde{n} u$ ndaa para Texupan. Posiblemente, ñut o "pueblo, lugar en donde existe algo" y sandaa o color "azul". Fray Francisco de Alvarado (1593) distingue entre "turquesa da color" que sería yusi y "color azul", en mixteco, sandaa. Al parecer, el motivo "turquesa" alude al color 
sandaa en el topónimo de Texupan y no a yuu yuchi o yusi (cf. M. E. Smith, 1973). Hasta donde sé, los signos tòponímicos de Texupan se asocian a una corriente de agua que M. E. Smith interpreta como una indicación de altepetl o pueblo. Sin embargo, ningún topónimo conocido de Acatlán la incorpora en su signo. Por lo anterior, es probable que A. Caso (1977) tuviera razón al suponer que algunos de los lugares representados en el Códice Nuttall fueran signos toponímicos de Acatlán y no de Texupan. En uno de ellos, conocido como "Jade-Serpiente emplumada", se incorporó el motivo "jade" al signo del lugar, indicando yuu yuchi o "pie. dra preciosa", tanto turquesa como jade, y no el color yadza cuij, como ocurriría si pensáramos que se refiere al nombre mixteco de Texupan, $\tilde{n} u$ ndaa. Otros topónimos que Caso incluye como posibles representaciones de Acatlán, son "Montaña con la punta torcida", "Joya azul", "Templo de la turquesa" y "Templo de la turquesa-Quetzalcóatl", es probable que los últimos sean variantes de un lugar cuya representación contiene un motivo "turquesa" con valor yusi en su signo.

En el Tulane, un documento que seguramente proviene de la Mixteca Alta, aparece la fecha 7 Lagarto 12 Caña, 1167 según A. Caso (1977), y relacionados con ésta se encuentran doce personajes encabezados por 1 Zopilote "Tláloc". Todos reunidos en el lugar que Caso nombró "TecpanAgua" y que en el manuscrito antecede a la fecha 7 Lagarto 12 Pedernal en la cual 1 Lluvia parece ser el fundador de un linaje con control político en un lugar "Cerro del jade". Aunque el signo cumple con los elementos yucu y yuu yuchi, mismos que contiene el topónimo de Acatlán en el Códice Sánchez Solís, el que sean análogos y sea, el último, signo de Acatlán, pudiera intentarse esclarecer a partir de la información genealógica que cada manuscrito da en forma separada sobre el hijo (dzaya) del fundador de este linaje.

Estableciéndolo un tanto arbitrariamente como "ego", 9 Caña en el Tulane aparece como hijo de 1 Lluvia y de su esposa (tay nitnahandaha) 2 Muerte. 9 Caña parece casarse con 2 (?) Viento, sus suegros (dzutudzidzo), 1 Pedernal y 11 Movimiento, se asocian a un lugar "Templo del tigre". 9 Caña, llamado A 8(?) Caña "Macana florida" en el Códice Sánchez Solis y relacionado con un lugar acompañado por la glosa yoco yoxi dixaa parece haber estado casado con ? Viento "Turquesa", hija (dzayad$z e h e)$ de ? Pedernal y de 7 Movimiento "Sol de pedernal" del lugar "Cerro del tigre y el enrejado" (figs. 4 y 5). Así que se encuentra duplicada la información genealógica en ambos manuscritos.

8 (?) Caña "Macana florida" es probablemente el mismo que el Tulane consigna como 9 Caña, y ? Viento "Turquesa" quizás sea la llamada 2(?) 
Viento. Por otra parte, ? Pedernal y 7 Movimiento "Sol de pedernal" posiblemente son aquellos que el Códice Tulane llama 1 Pedernal y 11 Movimiento, "señores" del "Templo del tigre" en este último manuscrito, y de "Cerro del tigre y el enrejado" en el Códice Sánchez Solis. Probablemente, topónimos de un mismo lugar, así como "Cerro del jade" y "Cerro de la piedra preciosa" parecen ser signos equivalentes de Acatlán.

3. "Tay nisiyo nuu sindi; los señores mixtecos": parentesco y matrimonio en un antiguo linaje de Acatlán

El espacio geográfico concreto en el cual vivieron los tay nisiyo nuu sindi del linaje que nos disponemos a analizar y que resulta del estudio comparativo de estos dos antiguos documentos mixtecos, el Códice Sánchez Solis y el Tulane, por ahora sólo lo podemos aproximar. Acatlán, el pueblo colonial, y el lugar referido por los signos toponímicos que hemos discutido y que aquí reunimos bajo un solo nombre, yucu yusi tisaha, no necesariamente coinciden en ubicación. Al parecer, el topónimo representado por los pictogramas "Cerro del jade" y "Cerro de la piedra preciosa" designa un territorio donde una característica geográfica propia le define al ser evocada en el pictograma. Un territorio donde cierto linaje, cierto yaattnuhundi, tuvo el dominio del poder político en yucu yusi tisaha en torno del siglo XII de nuestra era, un linaje cuyo fundador parece ser 1 Lluvia.

La Relación Geográfica de Acatlán (PNE,V), menciona que por lo menos hacia 1581

el pueblo de Acatlan ques cabecera questa en la rreal Corona, tiene cinco lugarejos que le son subjetos que son: La estancia de Misquitepec. La estancia de Eloixtlahuacan. La estancia de Tehuicingo. La estancia de Xayacatlan. La Estancia de Huacaltepec (PNE,V:56).

Todos, en la región Nuiñe y enclavados en un territorio bajo donde casi en cualquier terreno a lo largo de los valles que forman los ríos es posible el cultivo irrigado. En contraste, sobre las colinas sólo puede esperarse levantar un excedente agrícola en tanto el terreno sea especialmente favorable. Los suelos son semiáridos $\mathrm{y}$, donde existe, el aluvión que los recubre es poco espeso. El característico clima caliente se acentúa durante la estación lluviosa del año, alcanzado temperaturas extremas cercanas a los $41^{\circ} \mathrm{C}$ y ocasionalmente menores de los $10^{\circ} \mathrm{C}$ en la madrugada. Duran- 
te el invierno es rara el agua de lluvia y en el año no excede los $580 \mathrm{~mm}$ de precipitación (S.R.H.). Un territorio donde la única vegetación posible es la del bosque bajo y espinoso que tiende a aglomerarse en las ribe. ras, como la del tío Acatlán y en las cuantiosas cañadas de la región.

En estrecha dependencia con los ríos, los antiguos linajes ñuiñe encontraron la etapa de los naandeye, de los códices mixtecos; donde los sistemas de parentesco y matrimonio quedaron documentados, época que corresponde a la fase arqueológica Natividad (1000-1521 dC.) de Nochixtlán, en la Mixteca Alta (R. Spores, 1972); durante la cual, suponemos que vivieron los descendientes de 1 Lluvia y el propio fundador del linaje real de yucu yusi tisaha.

El Códice Sánchez Solis se ocupa de ? Pedernal, llamado 1 Pedernal "Fuego" en el Códice Tulane (cf, diagramas), quien debió haber vivido cuando 1 Lluvia inicia un linaje en el lugar "Cerro del jade", según el propio Tulane en 7 Lagarto 12 Pedernal, 1180 (?) (A. Caso, 1977), ya que, mencionado como 1 Pedernal "Fuego", durante el año mixteco 12 Caña, 1167 (?) (A. Caso, 1977), aparece asociado con una escena de donde se deriva el linaje anterior. En el manuscrito de la Mixteca Baja, ? Pedernal es el cuarto señor en el linaje real fundado por 2 Lluvia "Tla" huizcalpantecuhtli" en "Cerro del tigre y el enrejado", probablemente "Templo del tigre" en el Tulane, lo que me permitió vincular las genealogías de los linajes reales de 1 Lluvia y 2 Lluvia "Tlahuizcalpantecuhtli" al comparar ambos documentos.

Ahora bien, los padres de la esposa de 10 (?) Pedernal, pariente de ? Pedernal, 10 Flor "Hacha de pedernal" y de la señora 13 Movimiento "Movimiento-Hueso", celebran su propio matrimonio en el año 12 Pedernal, 1232 (?) (A. Caso, 1977). Así que los padres de 10(?) Pedernal debieron vivir en "Cerro del tigre y el enrejado" en ese entonces. Considerando que la madre de 10 en ese (?) Pdernal, 13 Mono "Hombrecillo que nace del llano", había nacido en "Cerro de la piedra preciosa" o "Cerro del jade" y había casado con 4 Pedernal "Tláloc-Sol", séptimo señor en el linaje de 2 Lluvia "Tlahuizcalpantecuhtli", hemos observado que entre $1180(?)$ y $1232(?)$ pueden ser ubicados cuatro distintos jefes del linaje real en "Cerro del tigre y el enrejado", por lo que en el mismo lapso debieron sucederse tres descendientes de 1 Lluvia y el propio fundador del linaje en yucu yusi tisaha. Si prolongamos esta relación de cuatro jefes de linaje gobernando cada cincuenta y dos años, podremos estimar que el último señor en el linaje de 1 Lluvia habría vivido alrededor del año 1375 (?). Sin embargo, E. Rabin (en M. Gaxiola y M.C. Winter, 1980) y D. Kelley (1982) han destacado las inexactitudes que encierra 
este tipo de cálculo propuesto hace varios años por A. Caso (1977), así que la ubicación temporal del linaje de 1 Lluvia es sólo aproximada.

M. Fortes (1953), ha explicado al linaje como una asociación política primaria, en la cual los individuos mantienen toda posible relación en la sociedad a través del propio contexto del sistema de linaje. En un estudio reciente, I. Buchler, apoyada en Radcliffe-Brown, supone compuesto al grupo de linaje por los miembros vivos del linaje en un determinado momento y que, en su conjunto, adquieren una identidad corporativa. Al igual que los miembros de los linajes, los miembros de los clanes pueden reivindicar la descendencia común de un antepasado remoto pero, mientras que los miembros del linaje pueden enumerar todos los pasos generacionales que los separan del fundador del grupo, los miembros del clan no pueden hacerlo (I. Buchler, 1982).

Comparando algunos códices mixtecos y basados en lo anterior, hemos intentado construir el criterio de linaje real ñuiñe. Un grupo de parentesco estructurado unilinealmente al considerar en forma patrilineal la filiación de los miembros del grupo. Es decir, la línea de descendientes reconocidos por éste, pone en relación únicamente a los miembros masculinos del grupo. Un patrilinaje fundado por un varón y que incluye tanto a los miembros vivos como a los muertos. Como grupo de linaje, domina el poder político en un espacio geográfico concreto.

Durante el primer cuarto de nuestro siglo, W.H.R. Rivers habia ya notado los elementos principales de los sistemas patrilineal y matrilineal de filiación, los que pueden traducirse, siguiendo a I. Buchler (1982), como la transmisión de la propiedad, del oficio y del proceso de composición del grupo social. Estos elementos quedan reunidos en los linajes reales ñuiñe de 1 Lluvia y 2 Lluvia "Tlahuizcalpantecuhtli", y probablemente en otros antiguos linajes mixtecos, también estructurados como patrilinajes. Aunque hemos considerado la posibilidad de que los manuscritos contuvieran una información genealógica "falseada" como un medio de legitimar el poder político, tales intrusiones genealógicas se agregarían en concordancia con el sistema de parentesco reconocido. Alguien podría resultar miembro espurio de otro grupo de parentesco, pero siempre en un sistema patrilineal.

La característica elemental del sistema de matrimonio ñuiñe fue la exogamia, el buscar esposa fuera del grupo de parentesco, práctica seguida entre grupos patrilocales vecinos.

La forma patrilocal de residencia dentro de los pequeños valles ñuiñe, el uso preferente del territorio y el crecimiento del propio patrilinaje debió de provocar conflictos con grupos cercanos. En un intento por prevenir 
la guerra de todos contra todos, aparecieron las "alianzas matrimoniales". El dzutudzidzo, suegro, y la dzehedzidzo, suegra, no tardaron en aparecer dentro del marco de los esposos y pudo hablarse de yo'q dzatna ha quachindi, de aliarse en amistad (F. Alvarado, 1593).

Si estudiamos en forma comparativa el Códice Sánchez Solís, que se ocupa principalmente del lugar "Cerro del tigre y el enrejado", y el Tula$n e$, que entre otros lugares lo hace de "Cerro del jade", encontraremos una serie de "alianzas matrimoniales" realizadas entre miembros de los patrilinajes de 1 Lluvia y 2 Lluvia "Tlahuizcalpantecuhtli", así como matrimonios celebrados entre éstos y otros linajes vecinos (cf. diagramas).

Ahora bien, al parecer, durante el siglo XIII se estableció un estrecho vínculo entre estos dos lugares mediante continuas "alianzas matrimoniales" entre miembros de sus linajes reales. En el último cuarto del siglo, los patrilinajes de 1 Lluvia y 2 Lluvia "Tlahuizcalpantecuhtli" reforzaron su alianza con un sistema que no parece haber tenido precedente, el hoy conocido como matrimonio entre primos cruzados, en lo que suponemos que fue el momento de mayor inestabilidad política para ambos linajes.

Al iniciarse el último cuarto del siglo XIII, la posición política de los linajes de 1 Lluvia y 2 Lluvia "Tlahuizcalpantecuhtli" debió ser incierta. Es posible que entonces otro grupo patrilocal amenazara constantemente sus territorios, creándose una situación política inestable en los lugares ñuiñe. Alrededor del año 1260 (?) de nuestra era, sabemos que los linajes mencionados realizan "alianzas matrimoniales" simultáneas con un linaje real del lugar yucu ndaa yee o "Cerro del puro, verdadero o recto caracol" (cf. A. Reyes, 1953), probablemente incluido en lo que era el territorio novohispano de "Tequitziztepec del Rey" (18 $\left.{ }^{\circ} 5^{\prime} \mathrm{N} ., 97^{\circ} 43^{\prime} 0^{\prime \prime}\right)$, también en la Mixteca Baja (M.E. Smith, 1973 y A. Caso, 1977). En el Códice Sânchez Solis, acompaña a la representación del topónimo la glosa yoco taa hyi; lugar con el cual se encuentra relacionada 4 Viento "Mariposa de fuego" y de donde se muda para casarse con 13 Mono "Movimiento", noveno señor de "Cerro del tigre y el enrejado", del linaje de 2 Lluvia "Tlahuizcalpantecuhtli" (figs. 6 y 7). Simultáneamente, en yucu yusi tisaha ocurre otro matrimonio entre linajes reales. 8 Lagarto, sexto señor en el linaje de 1 Lluvia, se desposa con 11 a 13 Hierba, hija de 7 Lagartija, entonces señor de yucu ndaa yee (Códice Tulane y A. Caso, 1977), por lo que suponemos que ambas señoras fueran hermanas, acuhuniya (A. Reyes, 1593), ya que para celebrar "alianzas matrimoniales" con otros grupos de descendencia real, era necesario que fueran miembros del mismo linaje real de yucu ndaa yee (cf. diagramas).

La Relación Geográfica de Acatlán ( $\mathrm{PNE}, \mathrm{V})$, menciona que: 
Trayan guerra los deste pueblo con los del pueblo de Ycucar y con los de Tepexic que son sus conterminos; yban armados a la guerra con armas fechas de algodon que aca llaman "ichcahuipiles", con rodelas de cañas maçiças y duras, y macanas de madera fechas a manera de espadas con los filos de pedernales y de nauajas. Lleuatuan arcos de madera con sus flechas con puntas de nauajas e de pedernal. Peleauan en campo raso de campo a campo y algunas vezes se subian en cerros $y$ se foraleçian (PNE,V:61).

En otro lugar, explica que el pueblo de Petlaltzingo, que era del partido de Acatlán, se encuentra a tres leguas de su cabecera. Posee dos estancias que le son sujetas, la estancia de Tepexic y la de Temascalapa:

Tenian guerra con los del pueblo de Teccistepec, su comarcano, y con los del pueblo de Acatlan: peleauan en esquadrones donde se juntauan, en llanos o çerros, y lo mas hordinario era subirse a los çerros altos y alli fortificar sus aloxamientos con albarradas de piedra y fosas y desde alli se defendian con galgas y armas arrojadizas (PNE,V:71).

En la tan inestable Región Ñuiñe, bien pudo haber ocurrido una guerra entre yucu ndaa yee y los estrechamente vinculados yucu yusi tisaha y "Cerro del tigre y el enrejado" en algún momento de la segunda mitad del siglo XIII. Como un medio para disminuir las luchas aparecieron los "casamientos entre enemigos", resultando una "alianza matrimonial" que controlaba en forma triangular las relaciones políticas de los linajes reales. Sin embargo, el conflicto político probablemente continuó, a pesar de los primeros matrimonios entre linajes reales enemigos. Sabemos por el Códice Sánchez Solís, que 5 Casa "Tláloc-Pedernal", nacido de la anterior "alianza matrimonial" entre $Y u c u$ ndaa yee y "Cerro del tigre y el enrejado", reforzó la estrecha alianza existente entre yucu yusi tisaha y su grupo patrilocal, al desposarse con 2 Venado "Papagayo de turquesa", miembro del patrilinaje de 1 Lluvia. El hijo de ambos, 4 Mono "TortugaXiuhcoatl", en la siguiente generación, debió de acentuar con otra "alianza matrimonial" el vínculo existente entre los linajes de 1 Lluvia y 2 Lluvia "Tlahuizcalpantecuhtli". La nueva alianza, siguiendo la norma exogámica, se efectuó dentro de un sistema de matrimonio entre primos cruzados. Es decir, 4 Mono "Tortuga-Xiuhcoatl", encontró esposa entre las hijas del hermano de la madre (cf. R. Fox, 1980), 7 Mono, séptimo señor en el linaje de 1 Lluvia y su propio tío. Aunque parece haber sido el único caso de matrimonio entre primos cruzados de los linajes reales ñuiñe que hemos estudiado, es probable que no fuera una "técnica" inusitada en el antiguo ámbito mixteco. En la generación siguiente, una segunda "alianza 
matrimonial" entre yucu ndaa yee y "Cerro del tigre y el enrejado", quizá, pudo haber solucionado una contienda que para entonces cumplía más de medio siglo de apremiar a los antiguos linajes reales ñuiñe.

\section{4. "Yo'q dzatna ha quachindi; aliarse en amistad"}

En los entornos del actual pueblo de Acatlán de Osorio, Puebla, la antigua alianza matrimonial ñuine intentaba asegurar la continuidad de los linajes reales, quedando cumplida al desposarse hijos de jefes de distinto grupo de linaje. Al contraer parentesco por afinidad, la ahora esposa mudaba su residencia del propio grupo patrilocal y se disponía a vivir con el linaje de su marido en un nuevo territorio. Es probable que con el matrimonio diera comienzo una discreta relación de parentesco entre afines, tnuhu tnahandaa, diferente a la establecida entre consanguíneos, tiuhu tnaha, reforzándose la alianza con nuevos matrimonios a realizarse en forma intermitente (cf. F. Alvarado, 1593). El hijo varón, dzaya yee, primogénito, dzaya yevua, por lo general sucedía a su padre como jefe del grupo de linaje y adquiría el dominio del poder político sobre su ancestral territorio a la muerte del mismo (cf. F. Alvarado, 1593). En forma regular, el Códice Sánchez Solís menciona a las mujeres que ingresan al linaje real de 2 Lluvia "Tlahuizcalpantecuhtli" como afines, al concretarse una "alianza matrimonial" con otro grupo de parentesco (figs. 8 y 9). De idéntica forma, se ocupa de las mujeres criadas dentro de este linaje real de "Cerro del tigre y el enrejado" y que, por una "alianza matrimonial", fueron mudadas del grupo, al del esposo. En un solo caso, sabemos que se realizó un matrimonio que vinculó a dos grupos patrilocales, sin involucrar en la "alianza matrimonial" a un jefe del grupo de linaje. Alrededor de la primera mitad del siglo XIV, un hermano del entonces señor de "Cerro del tigre y el enrejado", 5 Serpiente "Aguila que cae", casó con 12 Agua "Joyã-Flecha" de un lugar "Maíz-Mano", celebrándose una "alianza matrimonial" que pocas veces se registró en los códices mixtecos. 


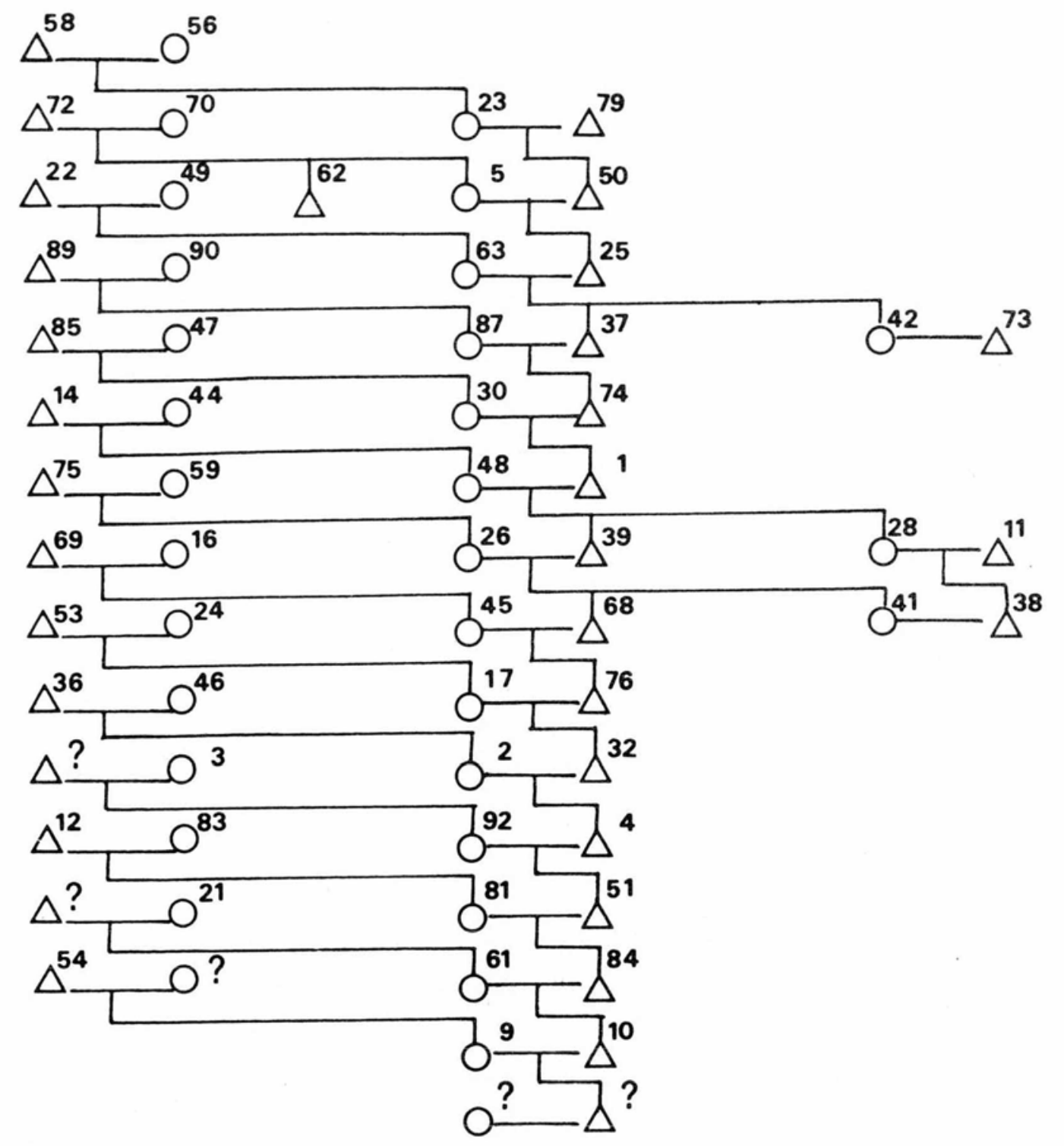

Figura 1. Diagrama del linaje fundado por 1 Lluvia en el lugar "Cerro del jade" o "Cerro de la piedra preciosa". Yucu yuи yuchi o Yucu yusi en el vocabulario de Fray Antonio de los Reyes. 


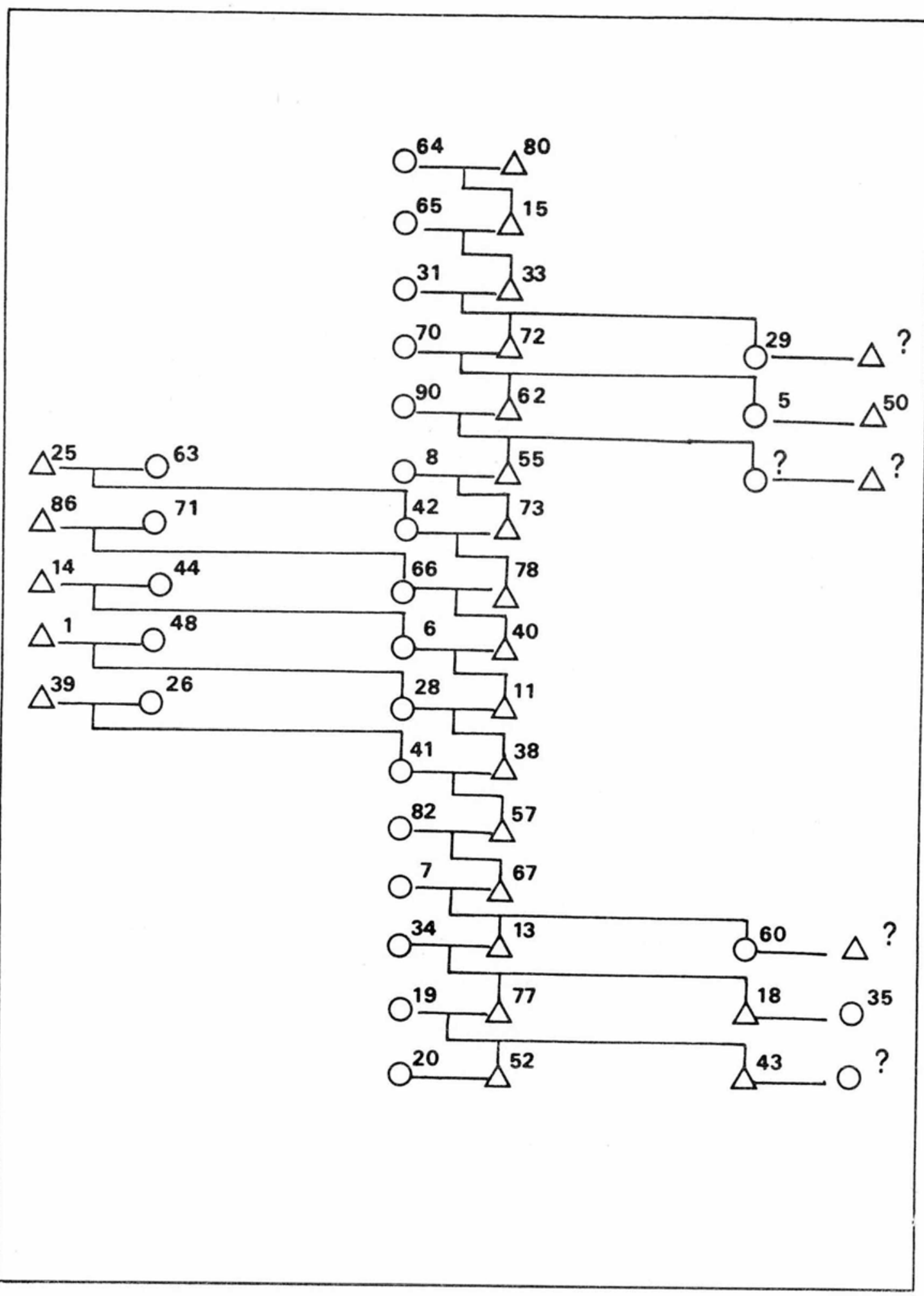

Figura 2. Diagrama del linaje fundado por 2 Lluvia "Tlahuizcalpantecuhtli" en el lugar "Cerro del tigre y el enrejado". Yucu ñaña yutnu cucua en el dialecto del vocabulario de Fray Francisco de Alvarado. 


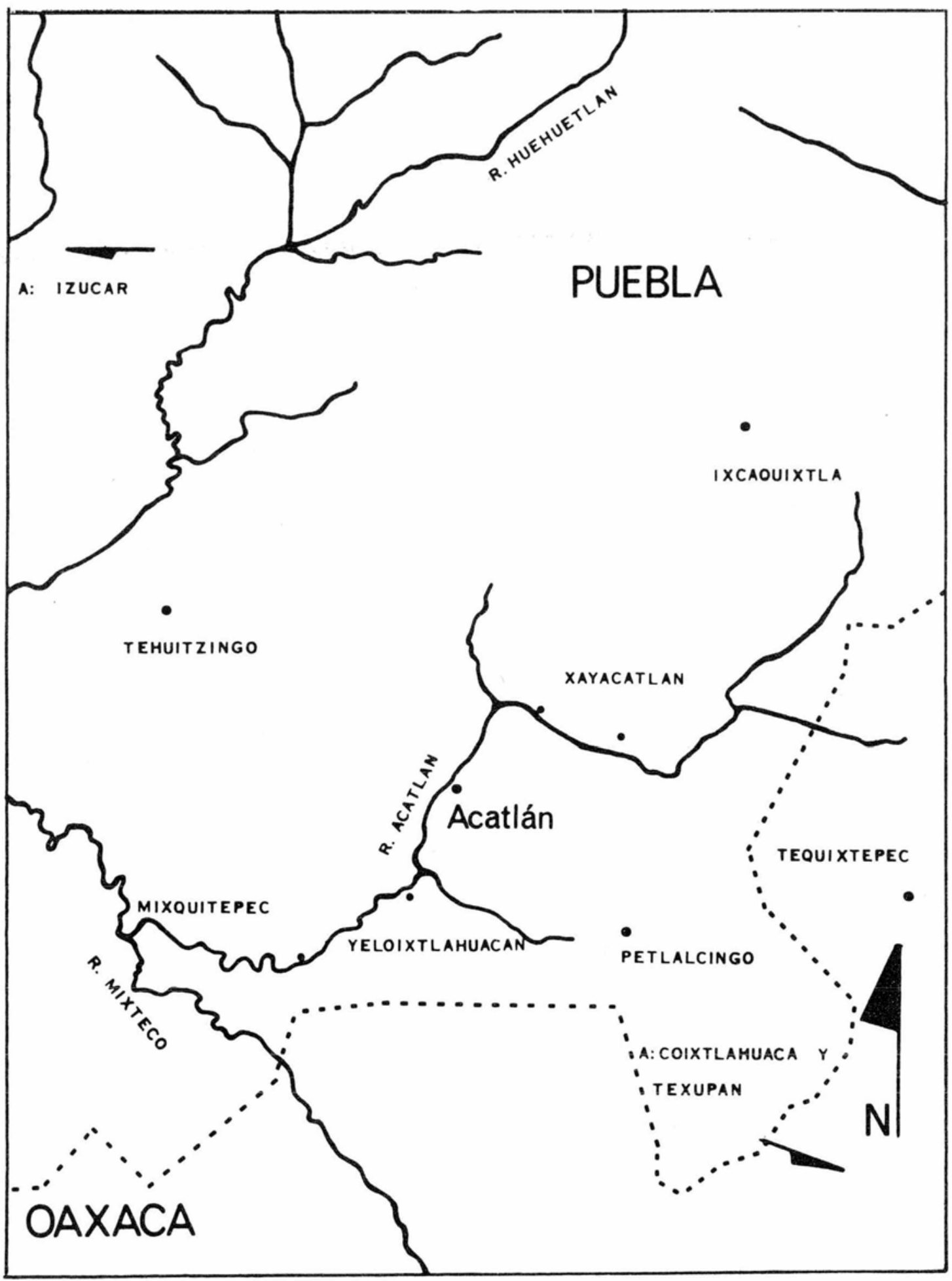

Figura 3. Una parte de la Región Ñuiñe. En las vecindades del actual poblado de Acatlán de Osorio, Puebla, debió encontrarse la antigua ciudad de 8 (?) Caña "Macana florida" y de su padre 1 Lluvia, fundador de un linaje real en "Cerro de la piedra preciosa". 


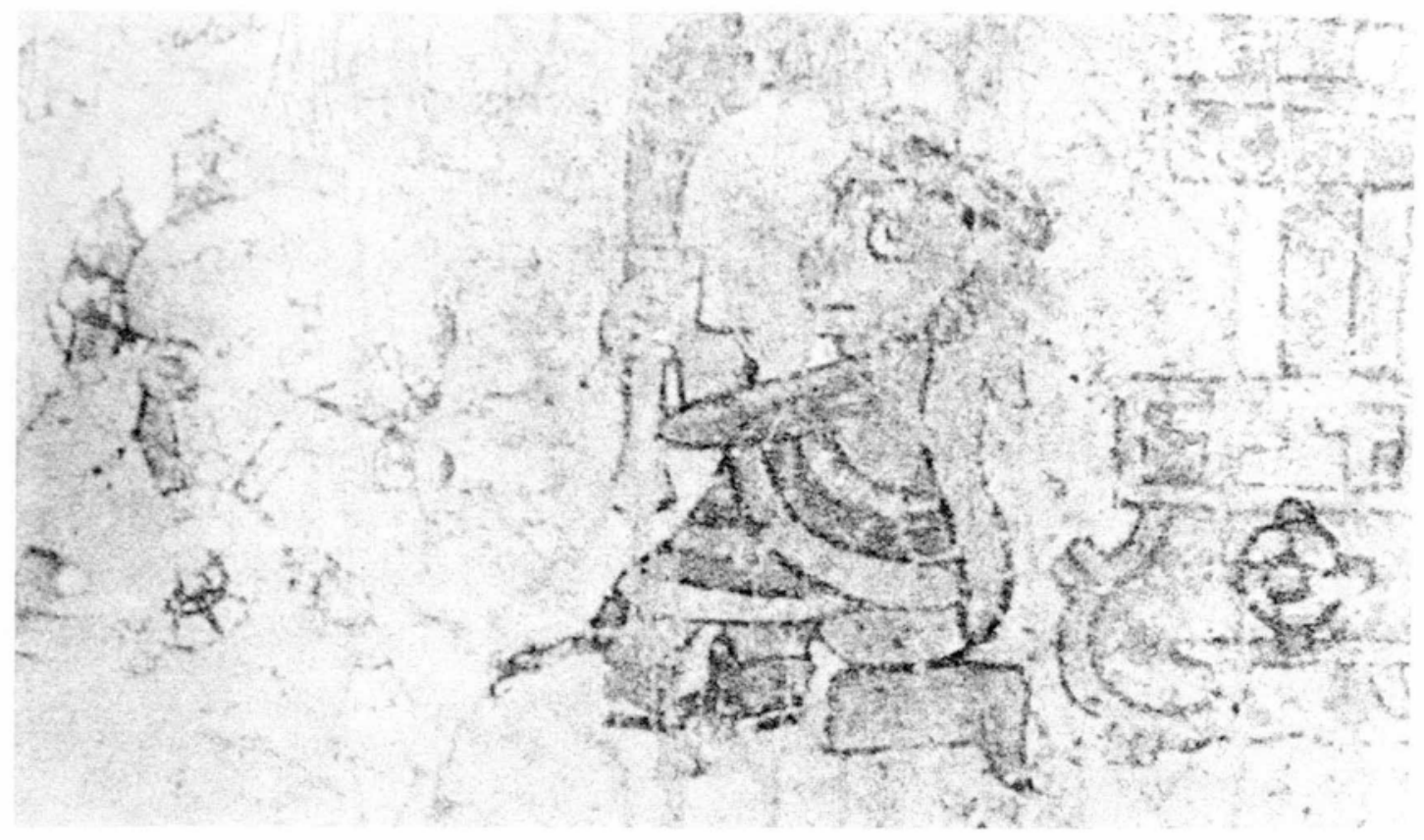

Figura 4. 8 (?) Caña "Macana florida" junto al topónimo de Acatlán, "Cerro de la piedra preciosa" o Yocoyoxidixaa, y su esposa? Viento "Joya de turquesa" de "Cerro del tigre y el enrejado" (Códice Sánchez Solís).

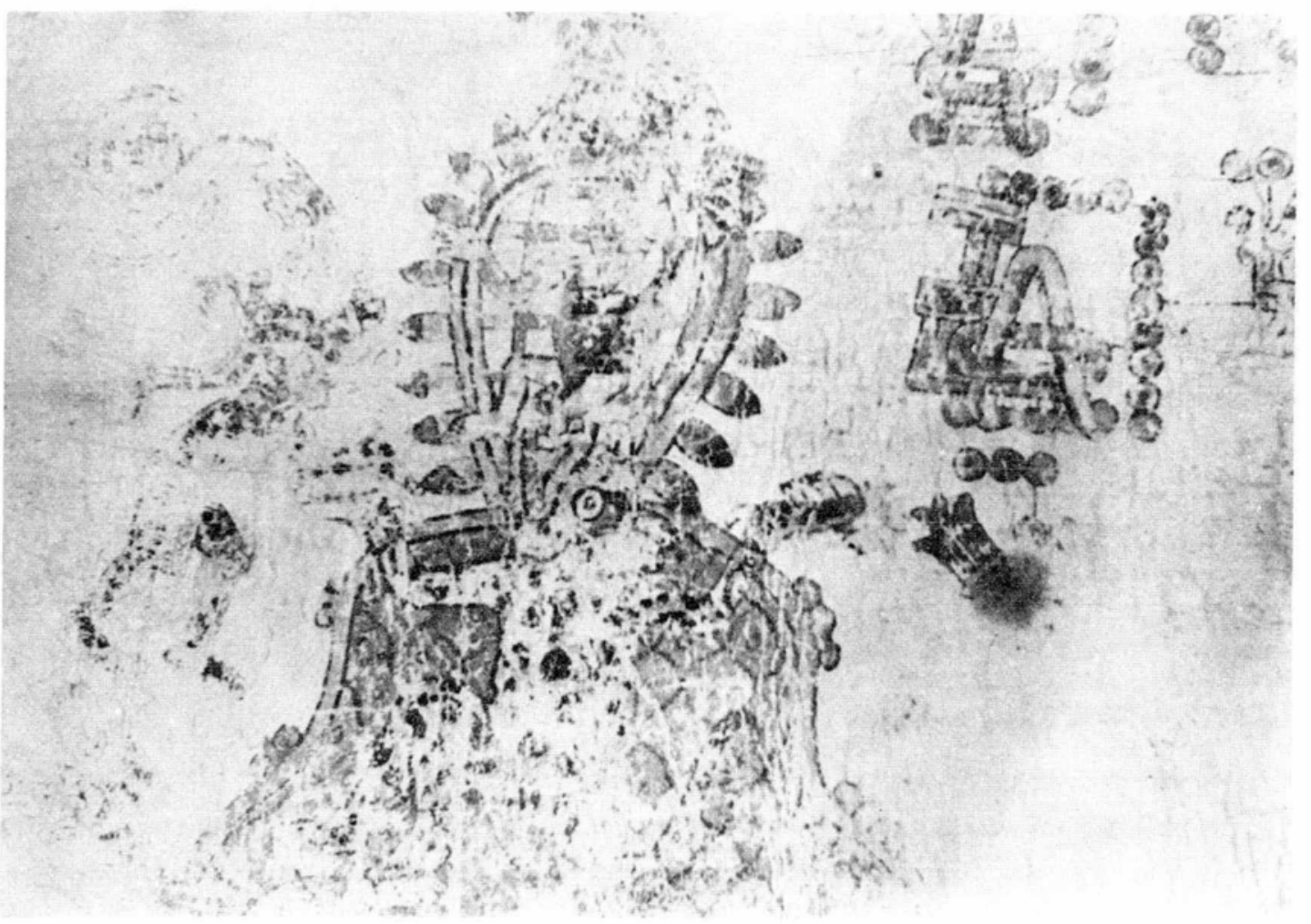

Figura 5. Signo del lugar "Cerro del tigre y el enrejado" (Códice Sánchez Solís). 


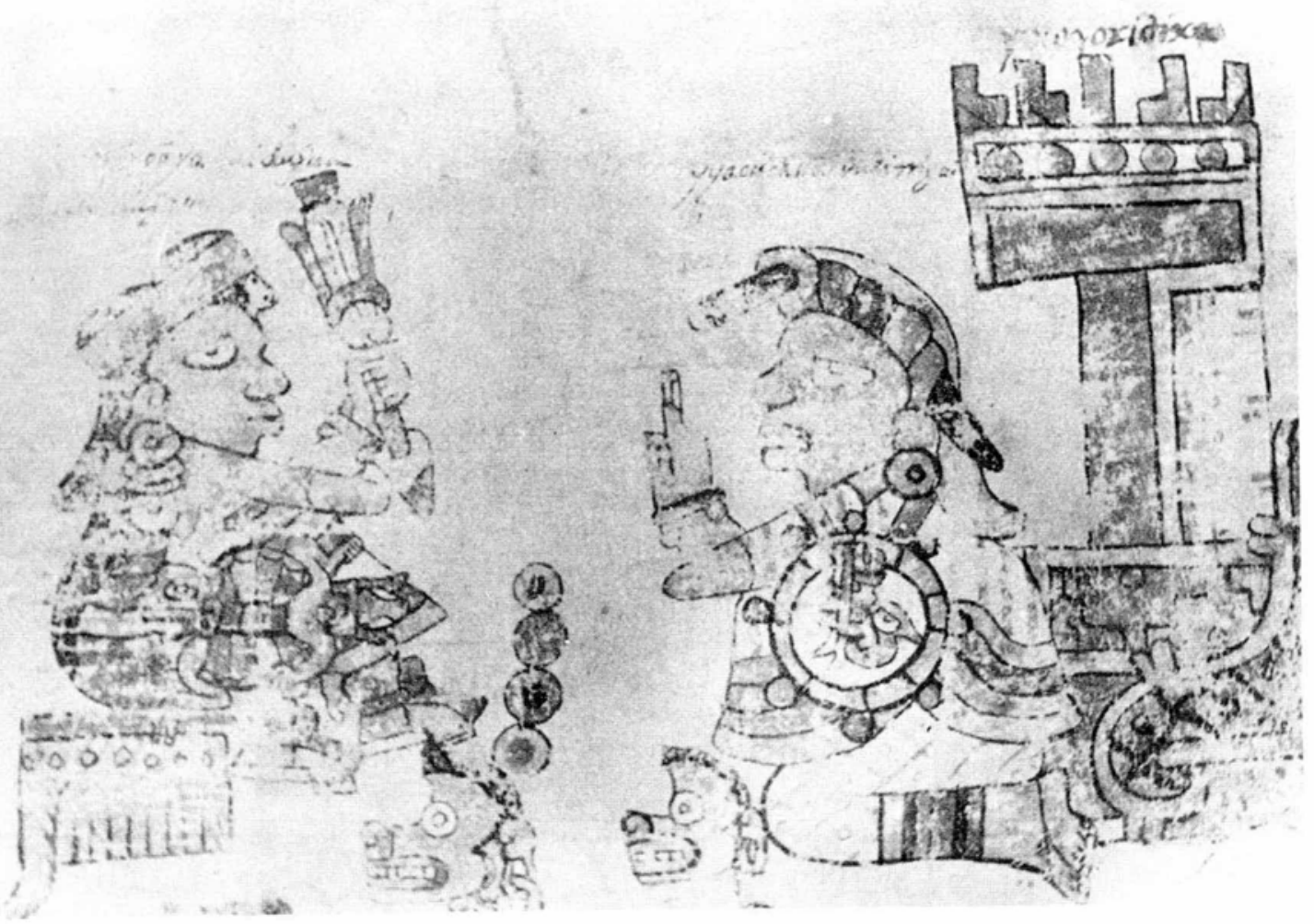

Figura 6. 4 Mono "Tortuga-Xiuhcoatl", señor de "Cerro del tigre y el enrejado", y su esposa 1 Mono "Joya de lagarto" de "Cerro de la piedra preciosa" o Yocoyoxidixaa (Códice Sánchez Solís). 


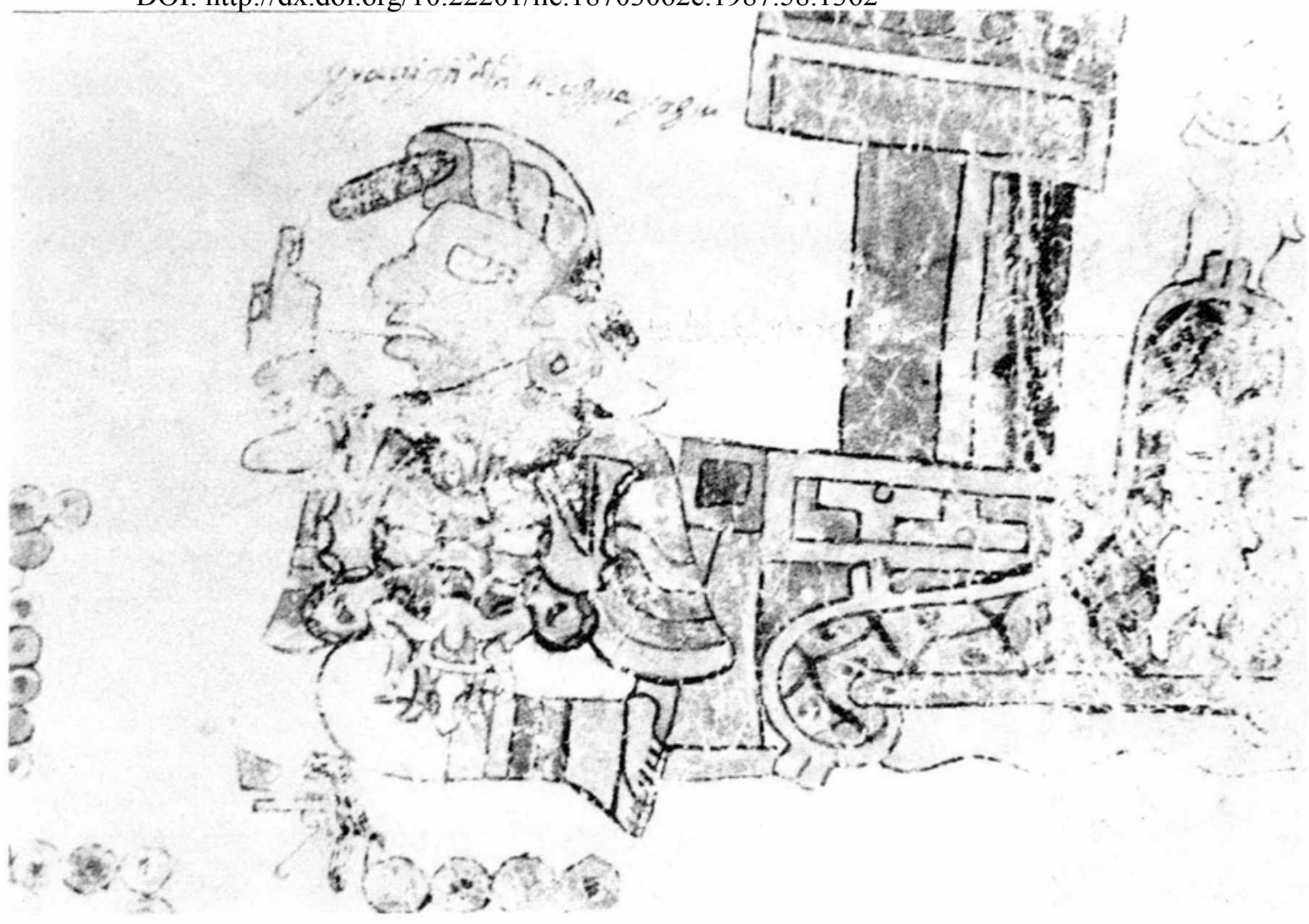

Figura 7. 4 Viento "Mariposa de fuego" junto al topónimo de Tequixtepec del Rey, "Cerro del puro, verdadero o recto caracol" o Yocotalahyi. Su esposo fue 13 Mono "Movimiento", señor de "Cerro del tigre y el enrejado" y abuelo de 4 Mono "Tortuga-Xiuhcoatl” (Códice Sánchez Solís). 
、 DOI: http://dx.doi.org/10.22201/iie.18703062e.1987.58.1362

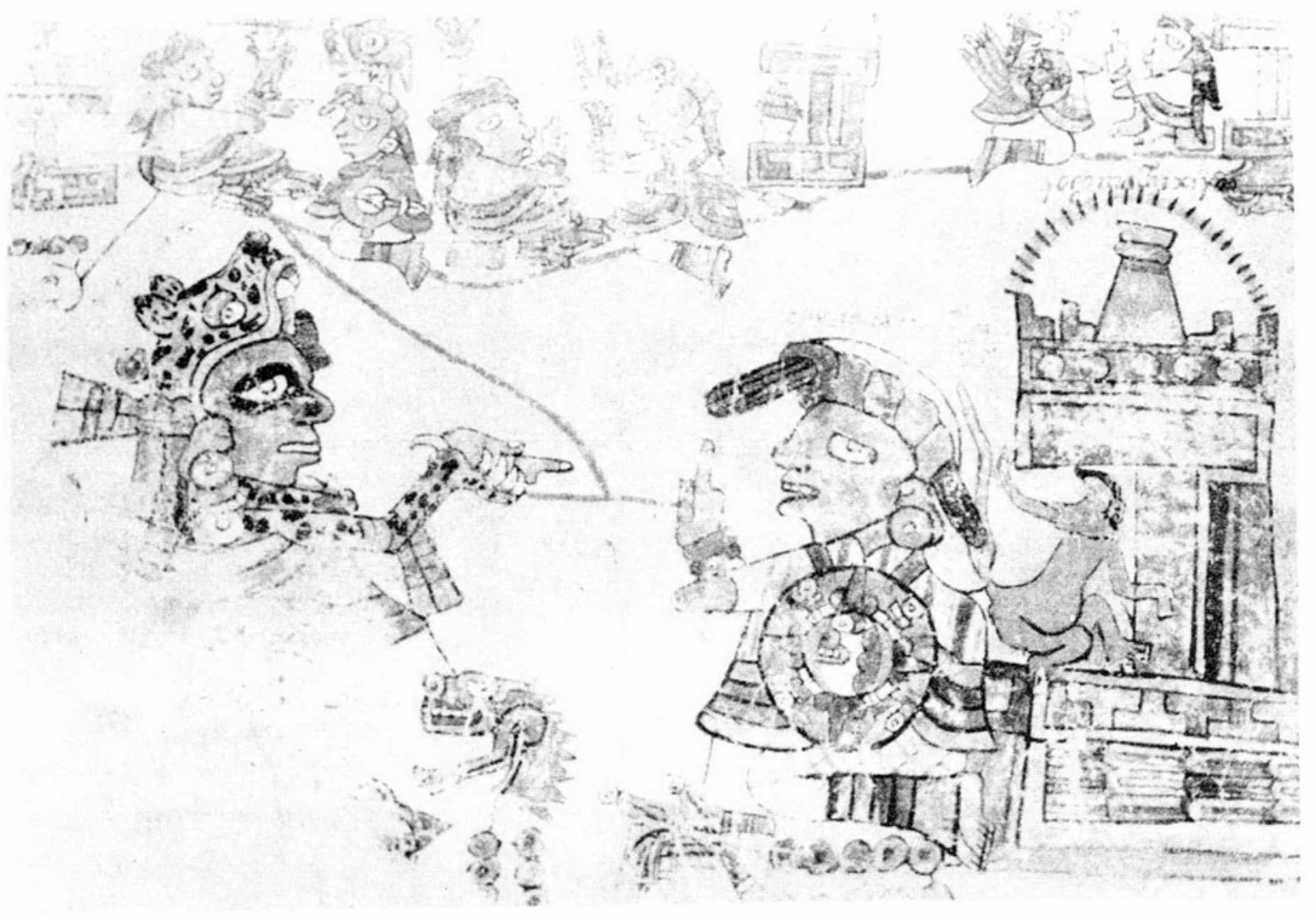

Figura 8. 10 Agua "Sol de pedernal” y su esposo 6 Lagartija "Tigre”, señor de "Cerro del tigre y el enrejado" (Códice Sánchez Solís). 
DOI: http://dx.doi.org/10.22201/iie.18703062e.1987.58.1362
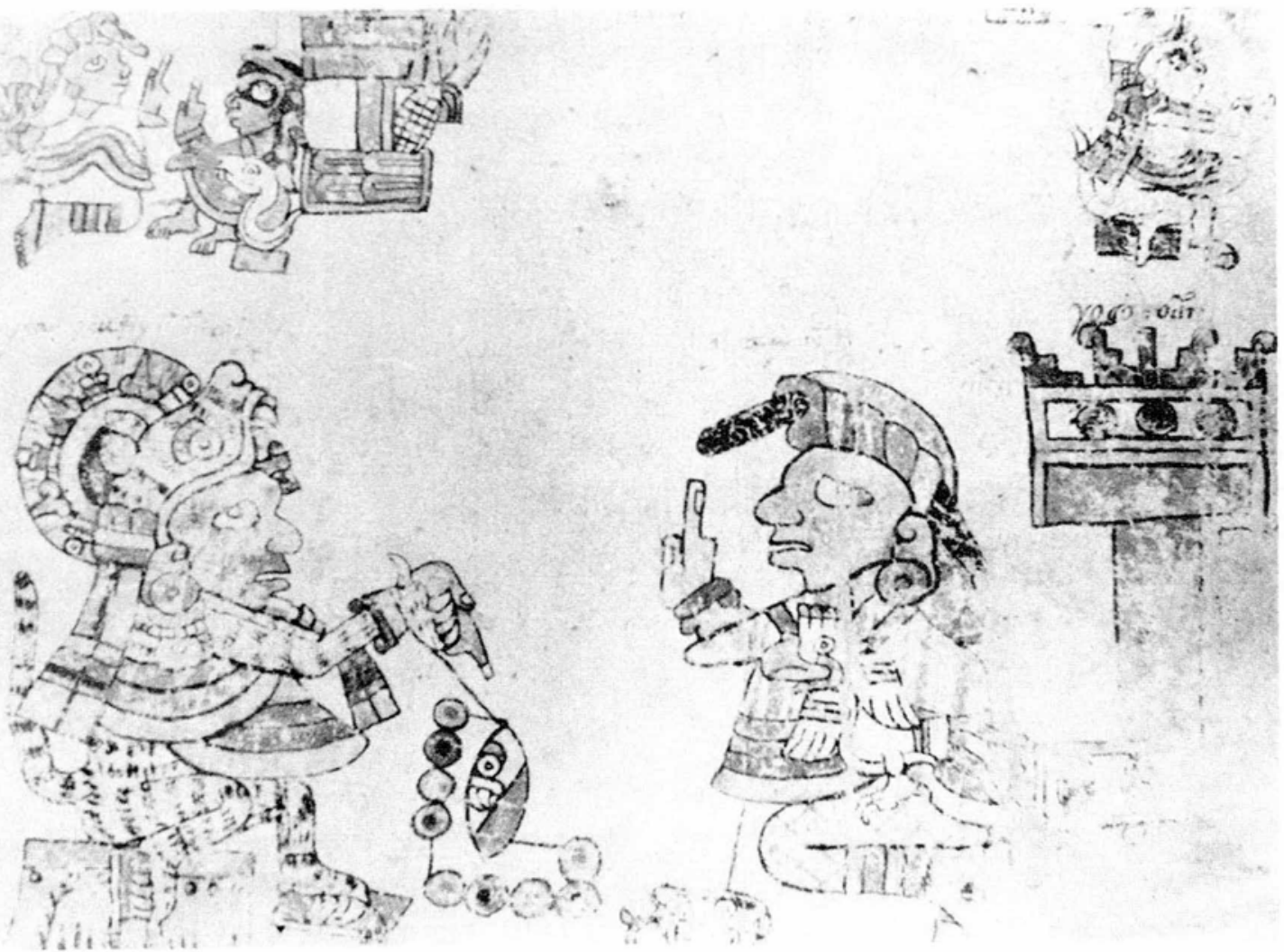

Figura 9. 9 Pedernal "Coyote-Sol", hijo de 10 Agua "Sol de pedernal" y 6 Lagartija "Tigre" de "Cerro del tigre y el enrejado", y su esposa 5 (?) Serpiente "Ave blanca" (Códice Sánchez Solís) . 


\section{CATÁLOGO DE PERSONAS ÑUIÑE INCLUIDAS EN LOS DIAGRAMAS*}

\section{LAGARTO}

Hombres

1. 8 Lagarto (C. Tulane)

\section{Mujeres}

2. 5 Lagarto (C. Tulane)

3. 13 Lagarto (C. Tulane)

\section{VIENTO}

Hombres

4. 4 Viento (C. Tulane)

\section{Mujeres}

5. $2(?)$ Viento (C. Tulane)

? Viento "Toya de turquesa" (C. Sánchez Solís)

6. 4 Viento "Mariposa de fuego" (C. Sánchez Solis)

7. 8 Viento "Mariposa celeste" (C. Sánchez Solís)

8. ? Viento "Joya-Flor" (C. Sánchez Solis)

9. ? Viento (C. Tulane)

\section{CASA}

Hombres

10. 4 Casa (C. Tulane)

11. 5 Casa "Tláloc-Pedernal" (C. Sánchez Solis)

12. ? Casa (C. Tulane)

\section{LAGARTIJA}

\section{Hombres}

13. 6 Lagartija "Tigre" (C. Sánchez Solís)

14. 7 Lagartija ( $C_{\text {. }}$ Tulane)

15. 10 Lagartija "Sol-Xiuhcóat1" (C. Sánchez Solis)

\section{Mujeres}

16. 4 Lagartija (C. Tulane)

17. ? Lagartija (C. Tulane)

* Los números que aparecen frente a los nombres de cada personaje indican su posición en los diagramas. 


\section{SERPIENTE}

Hombres

18. 5 Serpiente "Águila que cae" (C. Sánchez Solis)

\section{Mujeres}

19. 5 (?) Serpiente "Ave blanca" (C. Sánchez Solís)

20. 6 Serpiente "Sol de Xólotl" (C. Sánchez Solís)

21. 10 Serpiente (C. Tulane)

\section{MUERTE}

Hombres

22. 7 Muerte (C. Tulane)

Mujeres

23. 2 Muerte (C. Tulane)

24. 6 o 7 Muerte (C. Tulane)

\section{VENADO}

Hombres

25. 6 Venado (C. Tulane)

Mujeres

26. 1 Venado (C. Tulane)

27. 1 Venado "Pedernal-Cielo" (C. Sánchez Solis)

28. 2 Venado "Papagayo de turquesa" ( $C_{\text {. Sánchez Solís) }}$

29. ? Venado "Cráneo con quetzales (C. Sánchez Solis)

\section{CONEJO}

Mujeres

30. 1 Conejo (C. Tulane)

31. 2 Conejo (?) "Xiuhcóatl-Tortuga-Mariposa" (C. Sánchez Solis)

\section{AGUA}

Hombres

32. 6 Agua (C. Tulane)

33. 12(?) Agua "Garra de tigre" (C. Sánchez Solís)

\section{Mujeres}

34. 10 Agua "Sol de pedernal" (C. Sánchez Solis)

35. 12 Agua "Joya-Flecha” (C. Sánchez Solís) 
PERRO

Hombres

36. 1 merro (C. Tulane)

37. 4 Perro (C. Tulane)

\section{MONO}

\section{Hombres}

38. 4 Mono "Tortuga-Xiuhcóatl” (C. Sánchez Solís)

39. 7 Mono (C. Tulane)

40. 13 Mono "Movimiento" (M. Sánchez Solís)

\section{Mujeres}

41. 1 Mono "Joya de lagarto" (C. Sánchez Solis)

42. 13 Mono "Hombrecillo que nace del llano" (C. Sánchez Solis)

\section{HIERBA}

Hombres

43. 7 Hierba "Coyote blanco" (C. Sánchez Solis)

\section{Mujeres}

44. 1 Hierba (C. Tulane)

45. 6 Hierba (C. Tulane)

46. 7 Hierba (C. Tulane)

47. 10 Hierba (C. Tulane)

48. 11 a 13 Hierba (C. Tulane)

49. 12 o 13 Hierba (C. Tulane)

\section{CAÑA}

Hombres

50. 8 (?) Caña "Macana florida" (C. Sánchez Solís)

$$
9 \text { Caña (C. Tulane) }
$$

51. 10 Caña (C. Tulane)

52. 13 (?) Caña "Águila-Tlachtli" (C. Sänchez Solís)

\section{TIGRE}

\section{Hombres}

53. 1 Tigre (C. Tulane)

8 Tigre (Hueso 124, Tumba 7; Monte Albán)

54. 4 Tigre (C. Tulane)

55. 7 Tigre "Coyote sangriento" (C. Sánchez Solís)

Mujeres

56. 7 Tigre (C. Tulane) 


\section{ÁGUILA}

\section{Hombres}

57. 5 Águila "Aguila sangrienta" (C. Sánchez Solìs)

58. 11 Águila (C. Tulane)

\section{Mujeres}

59. 9 Águila ( $C$ Tulane)

60. 11 Ãguila (C. Sánchez Solís)

61. ? Águila (C. Tulane)

\section{ZOPILOTE}

\section{Hombres}

62. 3 (?) Zopilote "Venus-Tláloc" (C. Sánchez Solís)

\section{Mujeres}

63. 6 o 7 Zopilote (C. Tulane)

64. 7 Zopilote "Xólotl barbadö" (C. Sánchez Solís)

65. 10 Zopilote "Papagayo-Joya" (C. Sánchez Solis)

66. 13 Zopilote "Papagayo amarillo" (C. Sánchez Solís)

\section{MOVIMIENTO}

Hombres

67. 6 u 8 Movimiento "Insecto-Llamas" (C. Sánchez Solís)

68. Movimiento (C. Tulane)

69. 12 Movimiento (C. Tulane)

Mujeres

70. Movimiento "Sol de pedernal" (C. Sánchez Solís) 11 Movimiento (C. Tulane)

71. 13 Movimiento "Movimiento-Hueso" (C. Sánchez Solis)

\section{PEDERNAL}

Hombres

72. 1 Pedernal "Fuego" (C. Tulane)

? Pedernal (C. Sánchez Solis)

73. 4 Pedernal "Tláloc-Sol" (C. Sánchez Solis)

74. 8 Pedernal "Serpiente de fuego" (C. Tulane)

75. 8 Pedernal "Serpiente" (C. Tulane)

76. 9 Pedernal (C. Tulane)

77. 9 Pedernal "Coyote-Sol" (C. Sánchez Solis)

78. 10 (?) Pedernal "Lagarto" (C. Sánchez Solis) 


\section{LLUVIA}

\section{Hombres}

79. 1 Lluvia (C. Tulane)

80. 2 Lluvia "Tlahuizcalpantecuhtli" (C. Sánchez Solís)

\section{Mujeres}

81. 6 Lluvia (C. Tulane)

82. 12 Lluvia "Flor de tule" (C. Sánchez Soliss)

83. 13 Lluvia (C. Tulane)

\section{FLOR}

Hombres

84. 4 Flor (C. Tulane)

4 Flor "Coyote" (Hueso 124, Tumba 7; Monte Albán)

85. 6 Flor (C. Tulane)

86. 10 Flor "Hacha depedernal" (C. Sánchez Solis)

\section{Mujeres}

87. 13 Flor (?) (C. Tulane)

\section{DUDOSOS}

Hombres

88. ? ?"Xólotl" (C. Tulane)

\section{Mujeres}

89. ? ?"Casa-PLanta" (C. Tulane)

90. ? ?"Quechquémitl de petate" (C. Sánchez Solis)

91. 2 ? (C. Tulane)

\section{BIBLIOGRAFIA}

Alvarado, Francisco de. Véase Jiménez Moreno, Wigberto.

Balandier, Georges.

1976 Antropologia politica, Ediciones Península, Barcelona.

BUCHLER, IRA.

1982 Estudios de parentesco, Panorama de la antropología cultural contemporánea, No. 5, Editorial Anagrama, Barcelona.

CARNeiro, RoBert L.

1970 "A Theory of the Origin of the State", Science, 169:733-38. 
Caso, Alfonso.

1977 Reyes y reinos de la Mixteca, Fondo de Cultura Económica, México. FORTES, MEYER.

1953 "The Structure of Unilineal Descent Groups", American Anthropologist, 55:17-41.

FOX, ROBIN.

1980 Sistemas de parentesco y matrimonio, Alianza Universidad, No. 13, Alianza Editorial, Madrid.

Fried, Morton H.

1967 The Evolution of Political Society, Random House, New York.

Gaxiola, Margarita y Marcus C. Winier.

1980 "La sociedad mixteca de la época prehispánica", Revista Mexicana de Estudios Antropológicos, XXVI:81-93.

Jiménez Moreno, Wigberto, ed.

1962 Vocabulario en lengua mixteca por Fray Francisco de Alvarado [1593], reproducción facsimilar con un estudio de Wigberto Jiménez Moreno y un apéndice con un vocabulario sacado del Arte en lengua mixteca por Fray Antonio de los Reyes de Alfonso Caso, INI e INAH/SEP, México.

Kelley, David,

1982 "Relaciones tolteco-mixtecas y etnología tolteca". Ponencia presentada en el Simposio Área Maya Norte, Instituto de Investigaciones Antropológicas, UNAM, México.

PADDOCK, JoHN, ed.

1966 Ancient Oaxaca. Discoveries in Mexican Archaeology and History, Stanford University Press, Stanford, California.

Paso y Troncoso, Francisco del, ed.

1905 Papeles de Nueva España, Segunda serie, vols. IV y V. Madrid.

Reyes, Antonio de los Véase Jiménez Moreno, Wigberto.

SANCHEZ SOLIS, Códice.

1965 Burland, Cottie A., ed, Codex Egerton 2895, Graz, Austria (facsímil fotográfico a color).

SIERRA, Códice.

1933 León, Nicolás, ed, Códice Sierra, México (facsimil a color de una copia).

Simith, Mary Elizabeth.

1973 Picture Writing from Ancient Southern Mexico: Mixtec Place Signs and Maps, University of Oklahoma Press, Norman, Oklahoma.

SPORES, RONALD.

1972 An Archaeological Settlement Survey or the Nochixtlan Valle, Oaxaca, Vanderbilt University Publications in Anthropology, No. 1, Nashville, Tennessee. 
1976 "La estratificación social en la antigua sociedad mixteca", La estratificación social en la Mesoamérica prehispánica, P. Carrasco y J. Broda, eds., SEP-INAH, México.

TULANE, Códice.

University of Tulane, New Orleans (facsímil fotográfico).

WebB, MaLCOM C.

1973 "The Peten Maya Decline Viewed in the Perspective of the State Formation", The Classic Maya Collapse, T. Patrick Culbert, ed, University of New Mexico Press, Albuquerque. 